Creating collaboration. Advancing science. Impacting lives.

\title{
EXAMINING RELATIONSHIPS AMONG BODY IMAGE, SEXUALITY, AND SEXUAL FUNCTIONING IN WOMEN WITH CERVICAL AND ENDOMETRIAL CANCER
}

Christina M. Wilson, PhD, WHNP-BC 1 ; Deborah B. McGuire, PhD, RN, FAAN¹; Beth Rodgers, PhD, RN, FAAN¹; R.K. Elswick, PhD, NREMT-B ${ }^{1} ;$ \& Sarah M. Temkin, MD, Virginia Commonwealth University School of Nursing ${ }^{1}$

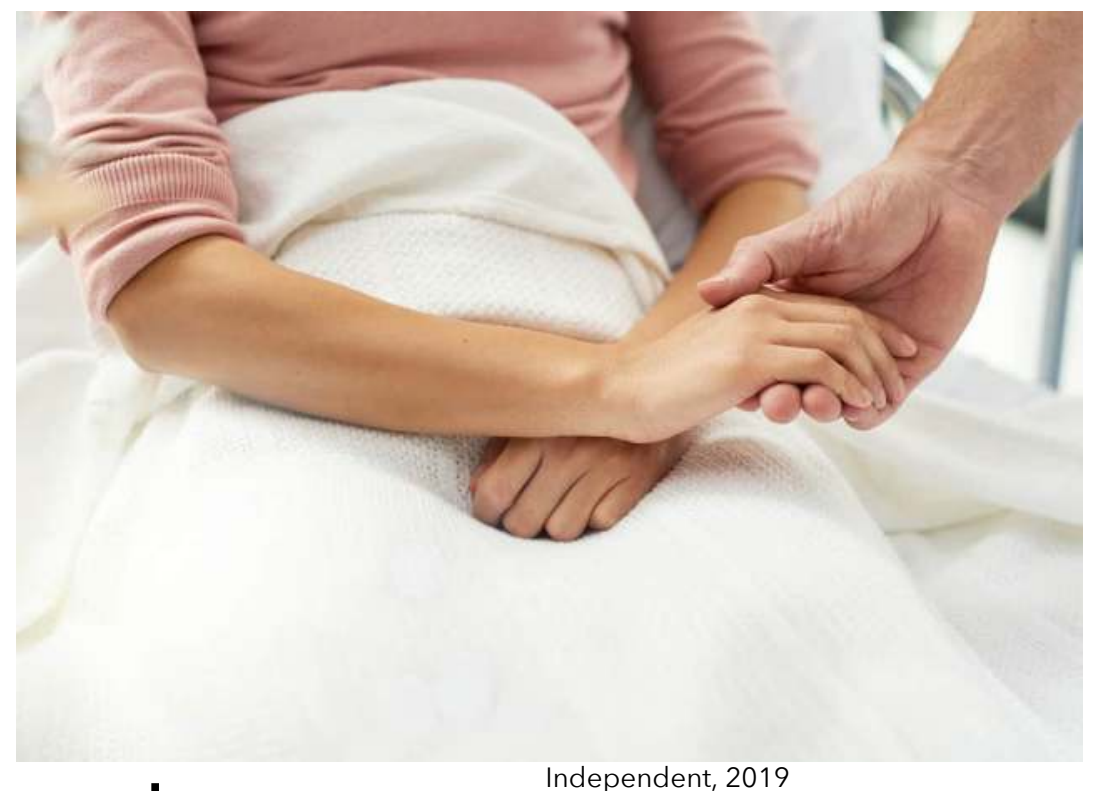

Background

- More than 56,000 new diagnoses of endometrial cancer and 13,000 of cervical cancer occur annually

- Women with these cancers experience significant alterations in body image, sexuality, and sexual functioning

\section{Study Aims}

- Examine relationships among body image, sexuality, and sexual functioning

- Understand whether cancer and/or other factors influenced women's views of concepts, and which were most important

\section{Methods}

- Non-experimental cross-sectional design

- $\mathrm{N}=27$ premenopausal adult women with stages I-III cervical or endometrial cancer, 3-36 months after completing treatment, and without major mental health issues

- Body Image Scale, Female Sexual Function Index, Female Sexuality Questionnaire, demographic form, open-ended items, and clinical data from medical chart

- Descriptive and correlational statistics, qualitative thematic analysis

\section{Results}

- Mean age was 40.3 years (range 27-56)

- Majority were White (55\%), Non-Hispanic (90\%), with some college education or more $(70 \%)$, in long term relationships $(65 \%)$, and with male partner preference $(90 \%)$

- Majority had cervical cancer (70\%), stage I disease (60\%), surgery $(60 \%)$, and radiation $(55 \%)$
- Significant relationships were identified between body image and sexuality ( $p=0.0244)$, and sexuality and sexual functioning ( $p<0.0003$ ), but not between body image and sexual functioning. Stages two and three disease were significantly associated with worse body image

- Women reported issues coded as body image, sexuality, sexual functioning, psychosocial, reproduction, and communication with healthcare providers/personal caregivers

\begin{tabular}{|c|c|c|}
\hline \multicolumn{3}{|c|}{$\begin{array}{l}\text { Qualitative Analysis of Women's Responses to Open-Ended Items with } \\
\text { Examples of Questions, Responses and Coded Categories }\end{array}$} \\
\hline $\begin{array}{c}\text { Open-Ended Item } \\
\text { (Frequency, Nature of } \\
\text { Responses) }\end{array}$ & $\begin{array}{l}\text { Example of Response(s) } \\
\text { (Nature) }\end{array}$ & $\begin{array}{l}\text { Coded Category } \\
\text { with Sub-Categories }\end{array}$ \\
\hline $\begin{array}{l}\text { O1 - How has cancer and } \\
\text { its treatment affected } \\
\text { your body image, } \\
\text { sexuality or sexual } \\
\text { function? } \\
\text { ( }=15 ; 1 \text { positive, } 12 \\
\text { negative, } 2 \text { neutral) }\end{array}$ & $\begin{array}{l}\text { "Treatment caused } \\
\text { menopause which has } \\
\text { affected my skin, hair, weight, } \\
\text { etc. I feel I have aged } 10 \\
\text { years since my treatment two } \\
\text { years ago. I wasn't prepared } \\
\text { for how I would look and feel } \\
\text { following treatment. It has } \\
\text { made me feel less desirable } \\
\text { and less interested in } \\
\text { pursuing relationships with } \\
\text { sexual partners." (negative) }\end{array}$ & $\begin{array}{l}\text { Body Image } \\
\text { Menopause } \\
\text { Sexual Functioning } \\
\text { Desire } \\
\text { Psychosocial }\end{array}$ \\
\hline $\begin{array}{l}\text { O5 - Tell me about your } \\
\text { communication with your } \\
\text { providers (doctors, } \\
\text { nurses, other) regarding } \\
\text { body image, sexuality } \\
\text { and sexual functioning. } \\
\text { Communicate with } \\
\text { healthcare providers? } \\
\text { (n=17; } 10 \text { yes, } 7 \text { no) }\end{array}$ & $\begin{array}{l}\text { "I feel like they didn't listen or } \\
\text { believe me; I felt gaslit } \\
\text { pertaining to my complaints } \\
\text { and symptoms with phrases } \\
\text { like "hmm that's uncommon" } \\
\text { or "oh no, sorry to hear about } \\
\text { sex." but then doing nothing } \\
\text { or confusing my symptoms." } \\
\text { (negative) }\end{array}$ & $\begin{array}{l}\text { Sexual Functioning } \\
\text { Communication } \\
\text { Question-asking } \\
\text { Listening }\end{array}$ \\
\hline $\begin{array}{l}\text { Of those who responded } \\
\text { yes, communication with } \\
\text { healthcare provider } \\
\text { (n=10; } 2 \text { positive, } 4 \\
\text { negative, } 4 \text { neutral) }\end{array}$ & & \\
\hline
\end{tabular}

Discussion

- One of the first studies to simultaneously examine relationships among body image, sexuality, and sexual functioning.

- Findings highlight the importance of examining relationships

- Future research should explore relationships with selected demographic and clinical variables, and communication issues with health care providers and personal caregivers

\begin{tabular}{|r|r|r|}
\hline \multicolumn{3}{|c|}{ Descriptive Statistics } \\
\hline$(\mathrm{n}=20)$ & Mean & $($ SD) \\
\hline Age (range = 27-56) & 40.3 & $(9.0)$ \\
\hline & & \\
\hline Race & $\mathrm{N}$ & $(\%)$ \\
\hline African American & 6 & $(30 \%)$ \\
\hline Asian & 1 & $(5 \%)$ \\
\hline White & 11 & $(55 \%)$ \\
\hline Other & 2 & $(10 \%)$ \\
\hline Ethnicity & & \\
\hline Hispanic or Latino & 2 & $(10 \%)$ \\
\hline Non-Hispanic or Latino & 18 & $(90 \%)$ \\
\hline
\end{tabular}

\begin{tabular}{|r|r|r|}
\hline Cancer Type & & \\
\hline Cervical & 14 & $(70 \%)$ \\
\hline Endometrial & 6 & $(30 \%)$ \\
\hline Stage & & \\
\hline 1 & 12 & $(60 \%)$ \\
\hline $1 I$ & 5 & $(25 \%)$ \\
\hline III & 3 & $(15 \%)$ \\
\hline HPV Status & & \\
\hline Positive & 7 & $(35 \%)$ \\
\hline Negative & 1 & $(5 \%)$ \\
\hline Not Available & 12 & $(60 \%)$ \\
\hline Surgery Type & & \\
\hline No Surgery & 8 & $(40 \%)$ \\
\hline Non-Radical & 5 & $(25 \%)$ \\
\hline Radical (laparotomy and lymph & 7 & $(35 \%)$ \\
node removal) & & \\
\hline
\end{tabular}

Research supported in part by Jonas Philanthropies and Sigma Theta Tau International Small Grant 